\title{
Publisher Correction: Integrated multi-omics framework of the plant response to jasmonic acid
}

Mark Zander, Mathew G. Lewsey (1), Natalie M. Clark, Lingling Yin (D), Anna Bartlett, J. Paola Saldierna Guzmán, Elizabeth Hann (1), Amber E. Langford, Bruce Jow, Aaron Wise, Joseph R. Nery ®D, Huaming Chen, Ziv Bar-Joseph (1), Justin W. Walley (1D, Roberto Solano (D) and Joseph R. Ecker (D)

Correction to: Nature Plants https://doi.org/10.1038/s41477-020-0605-7, published online 13 March 2020.

In the version of this Article originally published, the given name of the author J. Paola Saldierna Guzmán was spelt incorrectly as 'J. Paula Saldierna Guzmán'. This has now been corrected.

Published online: 21 July 2020

https://doi.org/10.1038/s41477-020-0743-y

() The Author(s), under exclusive licence to Springer Nature Limited 2020 